\title{
Failure Safety Method According to Battery's State-of Charge Condition in Electric Motorcycle
}

\author{
Tae-Hyung Lee and Byeong-Woo Kim \\ Dept. of Electrical Engineering, University of Ulsan, Ulsan, Korea \\ bywokim@ulsan.ac.kr,twinklelth@ulsan.ac.kr
}

\begin{abstract}
The present study proposes a failure safety method for an electric motorcycle according to the battery's state-of-charge (SOC) condition. We have reliably modeled an electric motorcycle by Model Based Design (MBD) using the Automotive Simulation Models (ASM) program. The Li-ion battery used in this model could have a serious problem if it discharges to $10 \%$ of the SOC. Therefore, the power supply should be limited before the SOC reaches the minimum level. At this time, the logic used to control the power of the electric motorcycle affects the driving distance and time. Various types of control logic are simulated to determine the optimum control logic. Accordingly, we propose a suitable failure safety logic that achieves good driving distance and time as well as better dynamic characteristics
\end{abstract}

Keywords: Automotive Simulation Models (ASM), Simulink, Electric motorcycle, In-wheel motor, Control logic, Battery Management System (BMS), Model Based Design (MBD), State-of-Charge (SOC), Failure safety

\section{Introduction}

In recent years, environmental and energy problems have emerged as important global issues. Among attempts to mitigate these problems, many studies worldwide have focused on electrically powered vehicles in the automobile industry. Some studies have focused on small electric cars for driving around in a city [1]. Unlike electric cars, electric motorcycles have not attracted much attention because of economic issues and a lack of relevant laws [2]. No studies have addressed the failure safety of motorcycles according to the battery's state-ofcharge (SOC) condition; studies have only focused on electric cars. Furthermore, studies have generally analyzed individual components in electrically powered vehicles, and not complete systems [3].

Unlike conventional vehicles with an internal combustion engine, electric vehicles are greatly affected by the characteristics of electric and electronic equipment such as the motor, battery, and inverter. In addition, with the increasing control requirements for electric and electronic components, it has become necessary to develop control methods to ensure the lifespan and safety of each component. Furthermore, it is necessary to analyze the vehicle behavior in terms of the vehicle system [3]. In particular, Li-ion batteries, which are the most widely used energy source in electric vehicles, are affected more significantly by charge/discharge characteristics than other batteries. When a Li-ion battery discharges to 
below the $2.7-\mathrm{V}$ minimum discharge voltage, its internal structure is destroyed, and this strongly affects its lifespan and safety [4]. Therefore, when the battery voltage drops below a designated value, the power drawn from it should be restricted to prevent damage to the battery while still being enough to move the vehicle to a safe area.

Accordingly, the present study develops a method for optimal failure safety logic in the designated SOC range (10-20\%) to protect the battery of an electric motorcycle; this method is based on an electric motorcycle model whose reliability has been verified in previous studies.

\section{System Design}

\subsection{Electric motorcycle model}

The electric motorcycle model was developed based on the specifications of a motorcycle with a 110-cc internal combustion engine, as listed in Table 1. To match the performance of a motorcycle with an internal combustion engine, a highly efficient Liion battery pack was installed along with a high-efficiency long-life battery management system to control it. In addition, to improve the durability and performance of the electric motorcycle, an in-wheel permanent magnet synchronous motor driving module with an embedded two-step transmission was used.

Table 1. Design specifications of electric motorcycle

\begin{tabular}{lcl}
\hline Parameter & Value & Unit \\
\hline Maximum rate speed & 80 & $\mathrm{~km} / \mathrm{h}$ \\
Cruising range & 70 & $\mathrm{~km}$ \\
(constant $50 \mathrm{~km} / \mathrm{h}$ ) & & \\
Capacity of battery pack & 2 & $\mathrm{kWh}$ \\
Maximum discharge rate & 5 & $\mathrm{C}$ \\
Power of motor & 4.88 & $\mathrm{~kW}$ \\
Nominal voltage & 73 & $\mathrm{~V}$ \\
Maximum weight & 285 & $\mathrm{~kg}$ \\
\hline
\end{tabular}

Figure 1 shows the electric motorcycle model developed using the model-based design (MBD) technique. For modeling the electric power components, the Automotive Simulation Models (ASM, dSPACE GmbH, Germany) program was used, and a vehicle kinetic model was developed using BikeSim (Mechanical Simulation Corp., USA). ASM and BikeSim are Open Simulink models for real-time simulation, which are third-party programs based on MATLAB/Simulink. Therefore, they are reliable and compatible with other Simulink programs for real-time simulations such as the hardware-in-the-loop simulation [4]. Figure 2 shows the flow of each component of the electric motorcycle model. The reliability of the electric motorcycle model used in this study was verified through a comparison between the vehicle test results and the simulation results. 


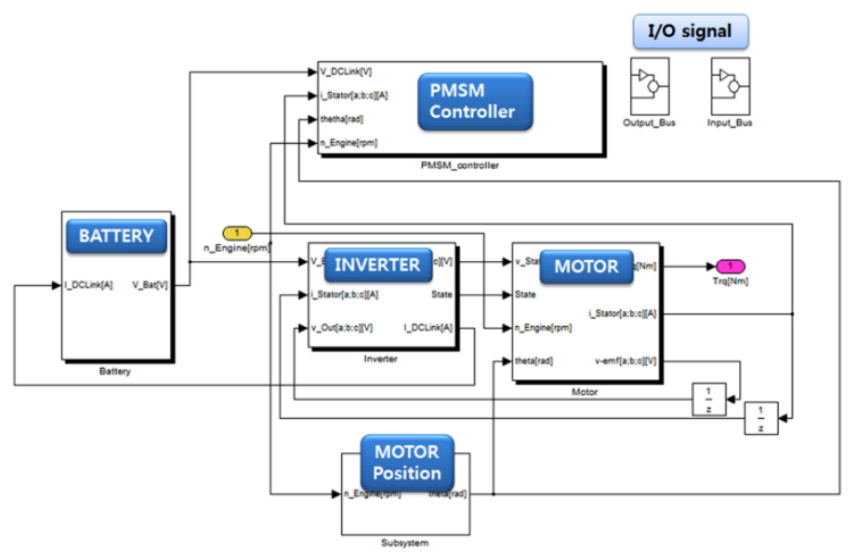

Figure 1. Component model of the electric motorcycle

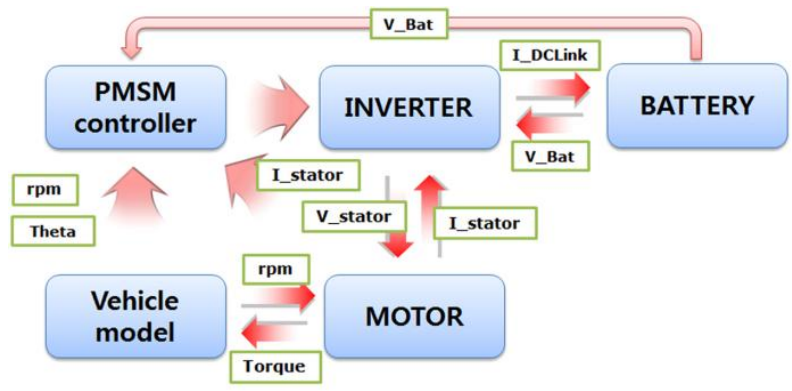

Figure 2. Control logic flowchart of the electric motorcycle

\subsection{Failure safety system configuration}

This chapter introduces the failure safety method according to the SOC of an electric motorcycle battery. As mentioned above, a Li-ion battery has higher efficiency than other batteries, but it is strongly affected by charge/discharge characteristics. Particularly, if it overdischarges to below the minimum discharge voltage $(2.7 \mathrm{~V})$, serious safety problems occur owing to the destruction of the internal structure of the battery. Therefore, for the electric motorcycle model used in this study, an integrated controller model was configured to protect the battery by restricting the power drawn from it. The integrated controller model was configured for step-by-step control using MATLAB's Simulink and Stateflow.

Figure 3 shows the discharge curve of the battery used in the electric motorcycle. A Li-ion battery has a different characteristic curve according to the discharge rate. As shown in Figure 3 , the voltage of the high-efficiency discharge curve differs from the basic discharge curve by $\Delta \mathrm{V}$.[6] Because the maximum discharge rate of the battery used in the electric motorcycle was $5 \mathrm{C}$, the failure safety logic of the integrated controller was designed based on the discharge curve for a discharge rate of $5 \mathrm{C}$. When discharging at $5 \mathrm{C}$, the discharge voltage drops to $2.7 \mathrm{~V}$ when the SOC drops to $10 \%$; therefore, restriction of the vehicle power begins when the SOC reaches $20 \%$ to secure sufficient time to warn the driver to move the vehicle to a safe place. In addition, when the SOC drops to $10 \%$, the power is cut off entirely to protect the battery, making it impossible to drive the vehicle. Accordingly, every simulation was carried out in the 10-20\% SOC range. To set the standard to restrict the power drawn from the battery, a simulation plan was made in the following order. 
- Step 1: Define the most efficient range of speed.

- Step 2: Define the most efficient type of control logic.

- Step 3: Define the safest type of control logic.

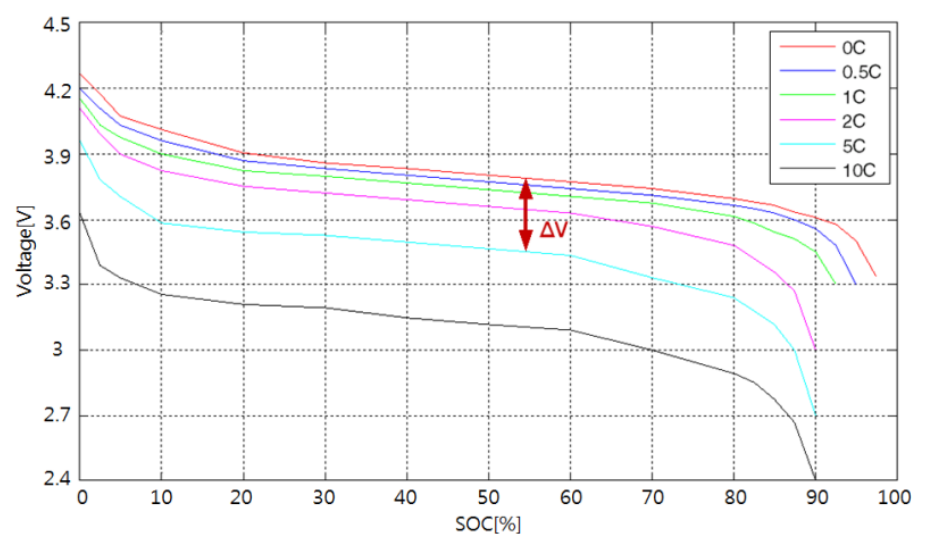

Figure 3. Discharge curve of the battery

\section{Simulation and Result}

\subsection{Real-time environment configuration}

The ds1006 and ds1401 modules of dSPACE were used to simulate the electric motorcycle model and the integrated controller model, respectively, in the real-time environment. These two models could exchange input/output values using controller area network communication.

Figure 4 shows the panel of the graphical user interface environment that is used to send commands to the electric motorcycle model and the integrated controller model through ControlDesk and to record the resulting values from them. The electric motorcycle simulation was carried out through this interface.

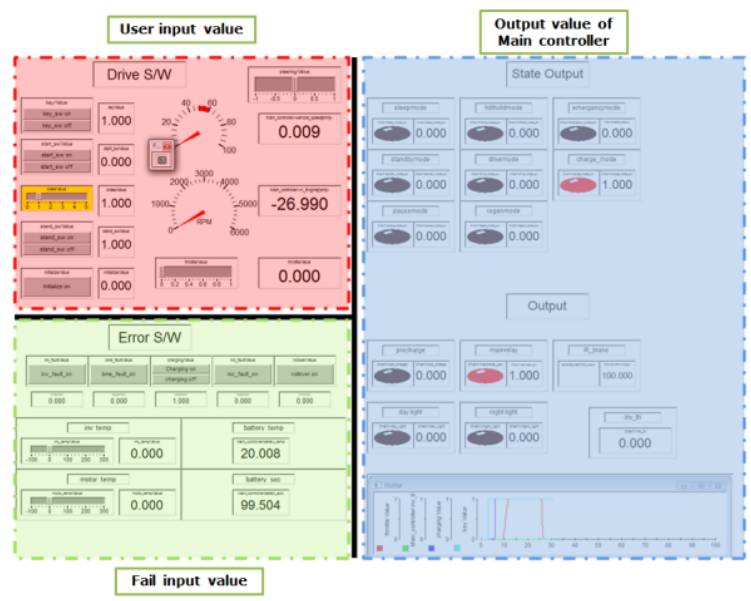

Figure 4. User interface of ControlDesk

\subsection{Simulation and result}


The road environment for the electric motorcycle simulation was modeled using BikeSim. The motorcycle was designed for riding around in a city; therefore, the simulation environment was made to comply with city road standards and Korean traffic laws [10]. The maximum speed of the electric motorcycle was set to $70 \mathrm{~km} / \mathrm{h}$, and accordingly, a road plane curve radius of $200 \mathrm{~m}$ designated for this speed was applied in the road environment. The electric motorcycle model was then ridden in a circular path with a radius of $200 \mathrm{~m}$.

When the battery SOC was below $20 \%$, the simulation reduced the speed to restrict the power consumption for an optimal drivable time and distance. For the simulation, the driving speed was set in the range of the minimum speed for stability $(20 \mathrm{~km} / \mathrm{h})$ to the maximum speed of the motorcycle $(70 \mathrm{~km} / \mathrm{h})$ in $10-\mathrm{km} / \mathrm{h}$ steps. Figure 5 shows the drivable time and distance at each test speed. As shown in Figure 5, the maximum difference in distance in response to the driving speed was $1000 \mathrm{~m}$, and the maximum difference in time was $700 \mathrm{~s}$. This result indicated that the most efficient speed range for drivable distance and time was $20-40 \mathrm{~km} / \mathrm{h}$. Therefore, when the battery SOC reached $20 \%$, the speed was restricted to $20-$ $40 \mathrm{~km} / \mathrm{h}$.

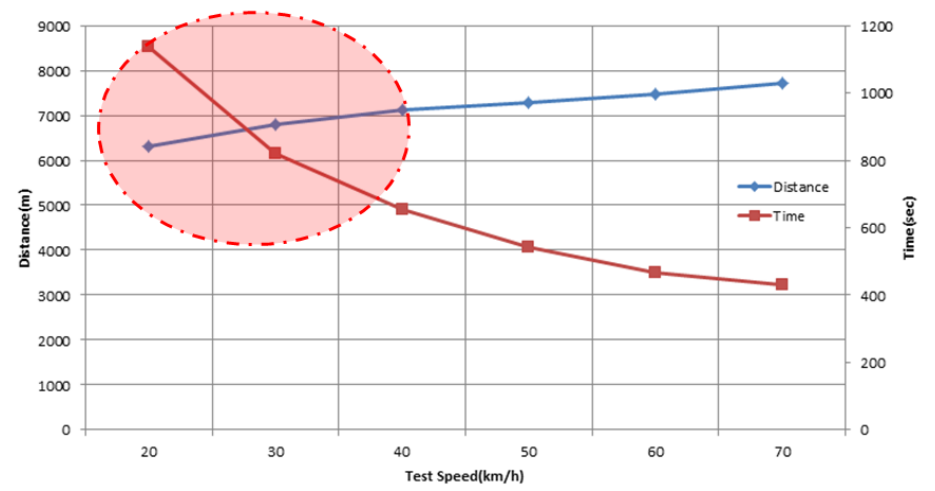

Figure 5. Graph of effective driving speed

Figure 6 shows five failure safety logics in the 10-20\% range of battery SOC. Because the speed range with the highest efficiency was identified in the simulation to be $20-40 \mathrm{~km} / \mathrm{h}$, the failure safety logic to restrict the power was configured using this speed range. Logic type 1 maintained the driving speed at $30 \mathrm{~km} / \mathrm{h}$ and had the best transportable distance and time efficiency. Type 2 was configured to reduce the motorcycle's speed linearly from the maximum $(70 \mathrm{~km} / \mathrm{h})$ to the minimum $(20 \mathrm{~km} / \mathrm{h})$ in the set speed range stated above. In type 4 , at the $15-20 \%$ range of battery SOC, the speed was linearly reduced from the maximum (40 $\mathrm{km} / \mathrm{h})$ to the minimum $(20 \mathrm{~km} / \mathrm{h})$ in the set speed range, and in the $10-15 \%$ range of battery SOC, a speed of $20 \mathrm{~km} / \mathrm{h}$ was maintained. In type 5 , the minimum speed $(20 \mathrm{~km} / \mathrm{h})$ of the set speed range was maintained continuously.

Figure 7 shows the simulation result for each type of failure safety logic, showing that types 3 and 4 best satisfied the requirements for maximum transport time and distance. Thorough this, it is shown that in the 10\%-20\% range of battery SOC, because the range to maintain a speed of $20 \mathrm{~km} / \mathrm{h}$ is longer, the transportable distance is shorter, but the operating time is longer. 


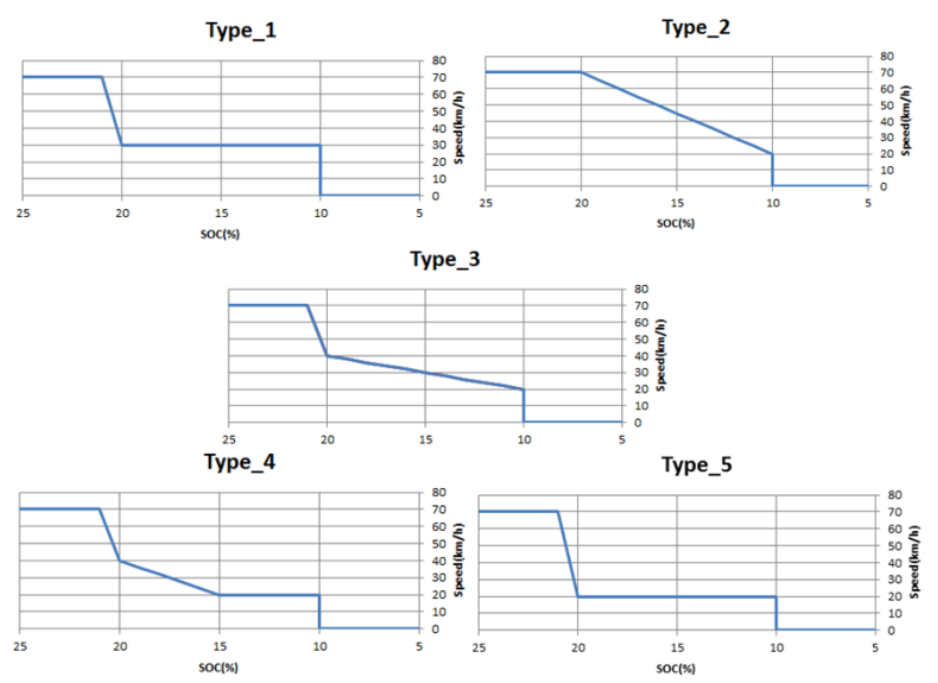

Figure 6. Types of failure safety logic

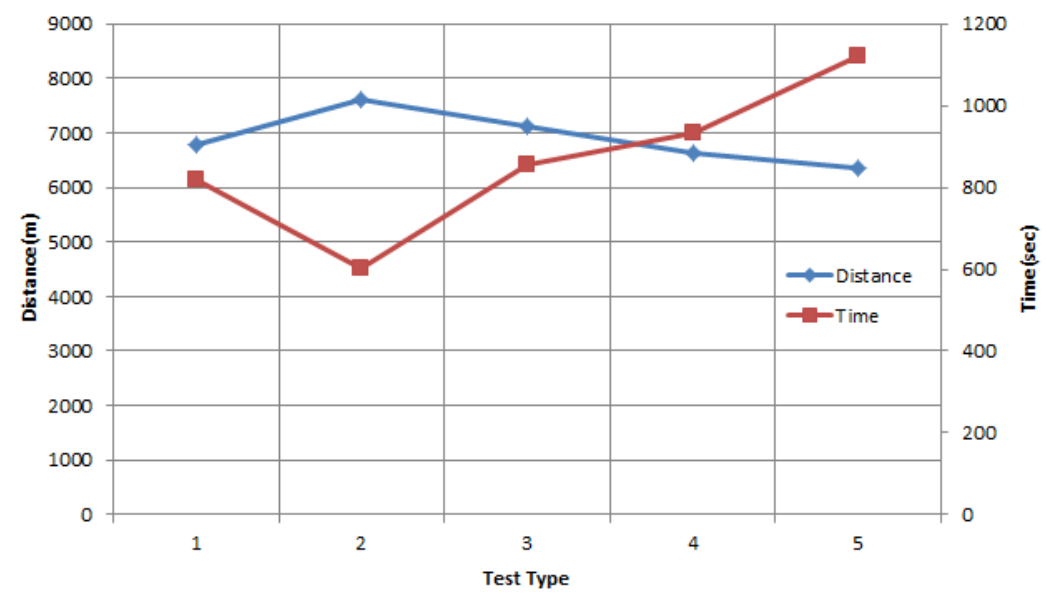

Figure 7. Distance and time result of each failure safety logic test

Figure 8 shows graphs that compare roll rates for types 3 and 4 selected based on the above result. When the battery SOC drops below the set limit, it is important for the driver to control the vehicle safely. In addition, because motorcycles have two wheels, unlike normal vehicles, the roll rate is an essential parameter for vehicle safety. Therefore, to identify the behavior of the motorcycle, two roll rates were compared. As shown in Figure 8, in both cases, the maximum roll rate was $8 \%$, but the roll rate of type 3 converges to zero without a radical change. Accordingly, it was determined that type 3 is the optimal failure safety logic for the motorcycle used in this study. 

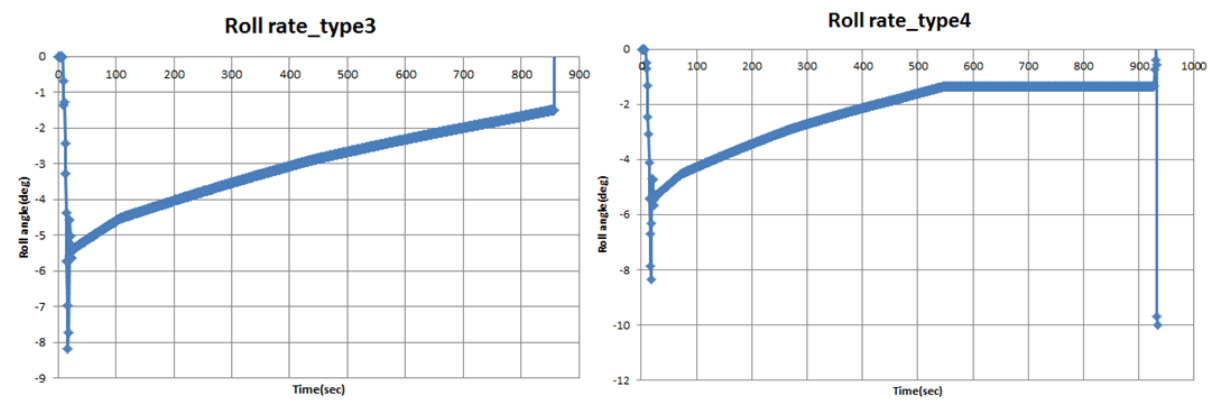

Figure 8. Graphs of roll rate results

\section{Conclusion}

In the present study, an optimal failure safety method was developed according to the battery SOC condition for an electric motorcycle model using MBD. To ensure the safety and lifespan of the battery, power was restricted to the 10-20\% range of SOC by an integrated controller; at this time, the failure safety logic was configured to provide sufficient time and distance so that the driver can reach a safe area.

In addition, the vehicle's kinetic behaviors under five types of failure safety logics were examined using BikeSim. The result showed that the failure safety logic that reduced the vehicle speed linearly from 40 to $20 \mathrm{~km} / \mathrm{h}$ in the battery SOC range described above afforded the greatest transport distance $(7,000 \mathrm{~m})$ and transport time $(820 \mathrm{~s})$. In addition, through a comparison of roll rates, a stable graph that converged without a radical change was obtained. Therefore, type 3 failure safety logic was identified to be the optimal method according to the battery SOC condition of the electric motorcycle.

The present study provides an optimal failure safety method according to the battery SOC condition of an electric motorcycle using MBD. In future studies, a failure safety method for key power components of the electric motorcycles, such as the motor and inverter, will be addressed.

\section{Acknowledgements}

This research was supported by the Ministry of Knowledge Economy (MKE), Korea, under the Convergence Information Technology Research Center (CITRC) support program (NIPA-2013H0401-13-1008) supervised by the National IT Industry Promotion Agency (NIPA).

\section{References}

[1] C. Meifen and J. Egashira, "High Efficiency Control of IPMSM for Electric Motorcycles", Power Electronics and Motion Control Conference, (2009), pp. 1893-1897.

[2] B. S. Kil, G. C. Kim, E. Lee and S. H. Oh, "The Analysis of an Electric Scooter's Performance through Motor and Battery Capacity Changing”, KSAE Annual Conference Proceedings, KSAE 09-A0533, (2009).

[3] J. K. Choi, "A Study on the Power Characteristics Analysis of Electric Motorcycle", Journal of The Korea Academia-Industrial Cooperation Society, vol. 12, no. 11, (2011), pp. 5156-5163.

[4] T. Lee, B. Kim and Y. Kim, "A Study on Power Characteristics Analysis of Electric Motorcycle using ASM", Journal of the KSAE, vol. 21, no. 3, (2013), pp. 58-65.

[5] C. S. Hearn, M. C. Lewis, R. C. Thompson and R. G. Longoria, "Modeling and Evaluation of a Plug-in Hybrid Fuel Cell Shuttle Bus", IEEE Vehicle Power and Propulsion Conference, VPPC '09, (2009), pp. 221228.

[6] ASM Electric Components Reference Manual, (2010). 
[7] P. Andrea, B. Beth, C. Robert, T. Pinak, R. Giorgio, G. Yann and M. M. Shawn, "Application of Modelbased Design Techniques for the Control Development and Optimization of a Hybrid-electric Vehicle", Proceedings of the Society of Automotive Engineers, (2009).

[8] Y. S. Kim, S. H. Lee, J. H. Jo, J. P. Hong and S. J. Kwon, "Development of Electric-drive-tractor Motor Controller", Journal of the Korea Institute of Electrical Engineering, (2012), pp. 18-20.

[9] D. G. Thomas, "Fundamentals of Vehicle Dynamics", SAE, US, (2002), pp. 112.

[10] Korea Ministry of Government Legislation, http://www.law.go.kr.

[11] T. -H. Lee and B. -W. Kim, "A Strategy of Fail Safety depends on Electric Motorcycle SOC Condition", Advanced Science and Technology Letters, Electrical Engineering, vol. 37, (2013), pp. 50-54.

\section{Authors}

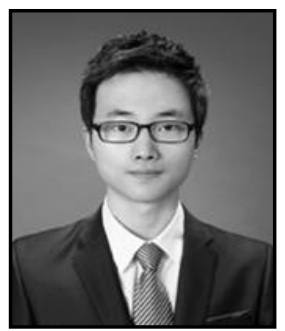

\section{Tae-Hyung Lee}

Tae-Hyung Lee received his B.S. degree in Mechanical \& Automotive Engineering from the University of Ulsan. Currently, he is completing his M.S. degree in Electrical \& Electronics Engineering at the University of Ulsan. His research interests include model based design, hardware in the loop simulation, and embedded system.

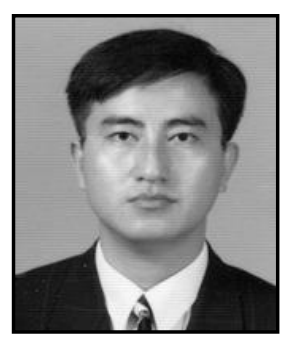

\section{Byeong-Woo Kim}

Byeong-Woo Kim received his B.S. and M.S. degrees and Ph.D. in Mechanical Engineering from the Hanyang University. He worked as a researcher at the KOSAKA R\&D Center in Japan and at the Korea Automotive Technology Institute from 1994 to 2006.

$\mathrm{He}$ is currently a Professor in Electrical \& Electronics Engineering at the University of Ulsan. His research interests include sensor fusion, $\mathrm{V} 2 \mathrm{~V}$ communication, embedded system, and software engineering, especially those aspects that are relevant to autonomous vehicles. 